\title{
Design of Shopping Guide System with Image Retrieval Based on Mobile Platform
}

\author{
Dibin Zhou, Baokun Hu, Qihui Wang, Bin Hu, \\ Leiping Jia, Yingfei Wu \\ College of International Service Engineering \\ Hangzhou Normal University \\ Hangzhou, China \\ e-mail: dibinz@zju.edu.cn
}

\author{
Lijun Xie \\ School of Aeronautics and Astronautics \\ Zhejiang University \\ Hangzhou, China \\ e-mail: zdxlj@zju.edu.cn
}

\begin{abstract}
Smartphone user group and rapid development of e-commerce, shopping guide system with image retrieval based on mobile platform is increasingly attracting attention. The portability and convenient camera feature of mobile phone allow mobile phone to have a natural advantage in e-commerce shopping. In order to adapt to the future trends of e-commerce, this paper studied mobile platform-based image retrieval technology and constructed an e-commerce-oriented shopping guide platform with commodity retrieval based on mobile devices. The platform supports the simple and convenient interaction methods and cutout design, and can provide effective retrieval results to make up for the deficiencies of the existing image retrieval system, thus has good practical value and significance of reference.
\end{abstract}

Keywords- Image Retrieval;E-commerce;Drag-drop Input; Mobile-based Cutout

\section{INTRODUCTION}

Currently, the global e-commerce trade volume continues rising, accounting for a growing proportion of the global economy and increasingly permeating all aspects of daily life. On the other hand, with the rapid development of computer technology, smartphone is more and more widely used, and its kernel has also changed from mono-core to dual-core, quad-core, and even multi-core, allowing it to be more functional. Transaction by phone becomes an important trend and tends to gradually replace the PC platform-based shopping. How to use the mobile platform to achieve fast and accurate shopping becomes an important trend of the future development of e-commerce technology.

Compared to the traditional PC platform, the smartphone platform has unique advantages and features in the electronic shopping, such as small size-based intelligence, strong computing ability, high resolution, portability; video capture feature and ordinary camera feature, allowing users to capture image data anywhere; physical characteristics: its physical uniqueness can support verification features, such as hardware verification and SMS verification, and support the positioning function.

On the other hand, the traditional e-commerce search is mainly based on keywords, thus has certain limitations, especially failure in accurate search in the case of lack of relevant information. Image-based retrieval technology can make up for some of the defects of the traditional keywordbased search that directly to find similar images according to the reference image. Currently, image retrieval technology has been greatly developed; in particular, the Internet giants are directly involved in the launch of related products, greatly promoting the rapid development of this technology and expanding the range of its application. E-commerceoriented image retrieval technology is the focus of attention and the main area of Internet applications, and the search friendliness, accuracy and high efficiency are the technical difficulties that are concerned about.

This paper designed and implemented a shopping guide prototype system with image retrieval based on mobile platform. Mainly for image retrieval in e-commerce, the system supports real-time camera search feature to achieve more accurate retrieval for shopping guide and effectively enhance the user experience. For the uneasy-to-operate characteristic of mobile platform, this paper studied dragand-drop image input method, and by analyzing the user interaction behavior provided a convenient image input function.

Meanwhile, taking into account that real-world images may be subject to interference of complex background, this paper studied mobile platform-based fast cutout feature to improve the existing algorithm, further improve the efficiency, and facilitate the quick search on mobile platform.

\section{RESEARCH BACKGROUND}

Image search technology[1] has been developing for over twenty years. The early systems were test-oriented or for industry-specific professional retrieval, such as digital image library, artifacts or face recognition, supporting retrieval of color, texture and shape. These systems included ADL system, Photofinder system, QBIC system, Virage system, MARS, Photobook, BDLP, Netra system, MetaSEEK, VisualSEEK, etc. Early image retrieval systems were technically immature, objectively constrained by hardware, and had limited range of applications.

With the rapid development of Internet technology and the exponential increase of multimedia data scale, image retrieval technology is developing rapidly. In terms of system efficiency, distributed design [2], MapReduce [3] architecture and multi-core technology $[4,5]$ have all been introduced to improve the storage and retrieval efficiency. On measure of system similarity, the various features of the 
image are primarily considered, including color features, texture features, structural features and spatial relationships. Thomas Deselaers [6] et al. made a detailed comparison of the image features and introduced the various evaluation feedback [7, 8] technologies into the image similarity evaluation to compensate for the defects of various feature similarity evaluation. Although there are various feature operators, they can hardly cover all the features, making it difficult to make substantive progress in image semantic understanding [9].

At present, the demand for image retrieval on the Internet is very active, becoming the core area of the next round of competition in the search field. According to the monitoring data of iUserTracker - a research system of iResearch on continuity of netizen behavior online, in the main vertical search categories, image search request reached 1.467 billion times in June 2011. Currently, many Internet companies have begun to focus on the design and application of image retrieval. For example, Google launches an image recognition feature Goggles. Baidu has also launched face retrieval service. Microsoft and Yahoo have launched similar products and services.

Image retrieval for e-commerce is an emerging field of research, which because of a huge commercial potential has gradually attracted attention. Some enterprises are turning to e-commerce-oriented image retrieval, such as Hitachi's GazoPa [10] system, and part of the systems have even extended to mobile platform [11] Image retrieval technology combined with commerce can better play its inherent advantages, mainly because:

Technically, commodity images generally have obvious image features and clear background, making it easy to calculate feature operators and make similarity comparison, thereby bypassing such core technical problems as image semantic, greatly improving retrieval accuracy;

From the market's perspective, the user demand is huge, and image retrieval can improve retrieval accuracy and service diversification, enhance the core competitiveness of enterprises and user shopping experience.

At present, image retrieval-based mobile shopping guide technology is a concern for many enterprises and hot spot in the market. Image retrieval can provide more accurate results to enhance user experience and improve search efficiency.

At the same time, mobile e-commerce is booming. Only in the Chinese market, the user scale of intelligent mobile platform is nearly 200 million. With the ongoing development of $3 \mathrm{G}$ services, mobile Internet access will become a new growth point to stimulate the continued growth of Internet users, while currently the mobile ecommerce is still in its infancy. Compared to other ecommerce platforms, mobile phone-based e-commerce has always some limitations, such as the too small screen, lack of keyboard or that the keyboard is too small, resulting in inefficient input of text and image.

Camera phone, on the other hand, has been widely popular. Seeing any product they like, users can take photos and search to quickly find the origins, manufacturers, and prices of similar products, as well as a variety of information on the product on the Internet. The combination of phone camera and shopping guide function will undoubtedly promote the development of e-commerce to a deeper level.

\section{FRAMEWORK DESIGN}

Mobile platform-based image retrieval system is based on CS architecture, including the server-side image retrieval engine and mobile-client interactive input.

The server is Soutugou search engine based on independent research and development, which applies parallel search architecture to support mega-scale commodity image retrieval, mainly used to search a variety of styles of shoes, bags and clothes, etc., and has a commodity data acquisition system with direct access to product data from large-scale commerce websites. The basic framework of the system is shown in Figure 1.

The search engine supports extraction of global, local and visual feature; the measurement of image similarity applies Nearest Neighbour Search(NNS) evaluation method of highdimensional data, i.e. the smaller the distance the higher the similarity; the system accelerates the retrieval through GPU cluster; the underlying is data storage, mainly including image data and the extracted feature data.

Mobile client inputs image data and feature options required by the search engine, such as color features and structural features. The image can have a variety of sources, such as local or network (supporting absolute and relative paths).

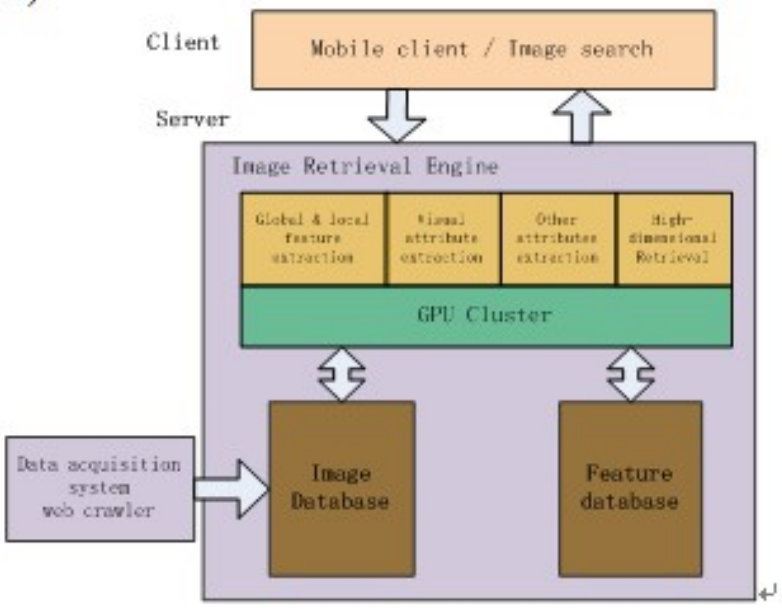

Figure 1. Framework of Mobile Image Retrieval System

\section{KEY TECHNOLOGIES}

Taking into account that the phone screen is small and uneasy to operate, and that mobile phone's computing power is generally lower than PC's, this paper studied drag-anddrop interaction method and quick cutout algorithm to improve the user experience.

The drag-and-drop image input:

For image retrieval on existing terminal, users often need to find the target image. It normally takes several times of interoperation to achieve the "Find" task. Web images are unable to be uploaded to browser for search. In order to facilitate the input of web images, it is often necessary to 
save the image first, and then search it, of which the process is too cumbersome. And taking into account that the phone screen is relatively small and the input is not convenient enough, this paper studied a drag-and-drop image input method [12]. The specific process is shown in Figure 2.

Before the user moves the mouse to drag the target image to the retrieval area of the search form, the user has to determine whether the operation meets the pre-set rules. If it does not meet the rules, the user will give up the above operation, and if it does, the user will determine whether the target image is from local or network. If the image is from the local, the user get the image from the local; if the image is from the network, the user will determine whether it is from a relative address or an absolute address. If the target image is from an absolute address, the user obtain the image from the network; if the target image is from a relative address, the user will according to the domain name and the relative address of the target image determine the absolute address, and then obtain the target image from the network based on the absolute address determined.

Given user habits in the operation, image input is to be determined when user behavior meets the following rules.

- When the mouse pointer is in the legitimate area of the target image, press the mouse button. The legitimate area of target image is the interior area within the external contour of the target image;

- When the mouse pointer dragging the target image is in the retrieval area of the search form, release the mouse button;

- When the moving distance of mouse pointer is greater than 0 .

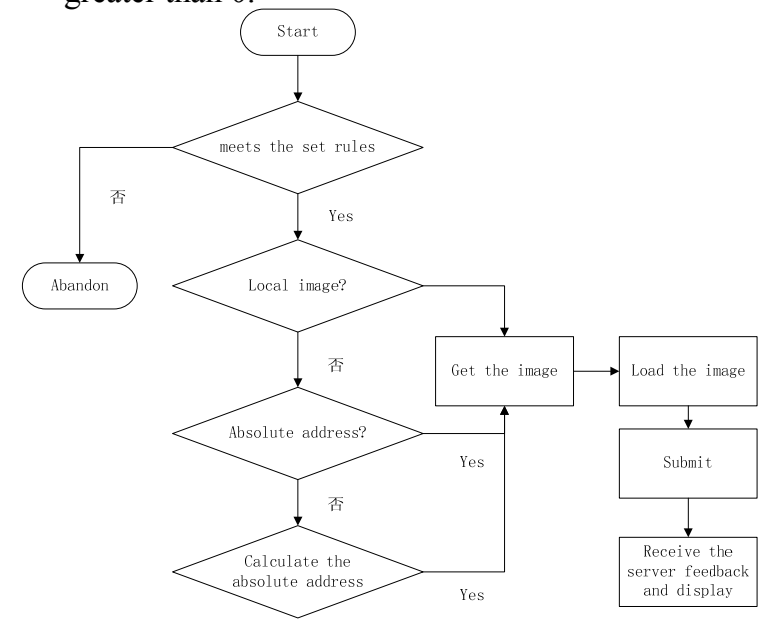

Figure 2. Drag-and-drop Input Process

Drag-and-drop input method saves the trouble of opening the file dialog box to select image or download image from a web page, which can significantly reduce the interactive operation, making the image input process simple and quick.

For a simple image, its features are relatively easy to extract and compare, but real-world images often have a complex background and are easily affected by light impact, making it difficult for the existing algorithms in the case of no interaction to intelligently extract target object, thus few practical retrieval systems support local feature retrieval. To this end, this paper specially designed a mobile platformbased cutout algorithm. The algorithm mainly based on the improved grabcut algorithm selects the appropriate target range through interaction with the user and by finding the method for maximum flow and minimum segmentation of undirected connected graph constituted by different areas achieves the best segmentation of the target area. On this basis, the high dimensional feature of the color, texture and shape, etc. are calculated, and encrypted and converted into 01 encoded byte stream, which is sent to the retrieval server. The server decrypts the feature codes received, uses parallel retrieval algorithm to obtain the corresponding subkeys to high dimensional indexing and return user the most similar commodity data. Figure 3 shows the process, which through outlining the foreground and background pattern achieves the separation of the foreground pattern and the background region. The images after treatment are uploaded to the image search engine, and the engine feeds back similar images.

For mobile platform with lower computing power, the process can be completed at the server side. The server's powerful computing capability can be used to achieve fast cutout, thereby avoiding the users waiting for too long.

\section{System Testing}

The system mainly provides retrieval of clothing, shoes and bags. Figure 3 is a screenshot of the camera phone search, showing the search results of clothing. All commodity images are sorted according to similarity, i.e. the more similar images are ranked more ahead.

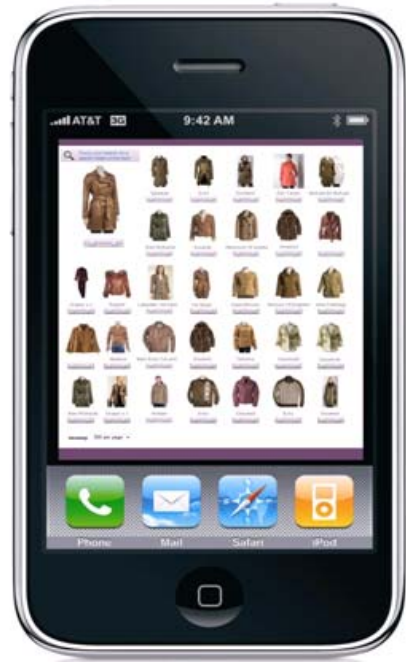

Figure 3. Retrieval of Image with Simple Background

Images with complex background must first be removed of the background, as shown in Figure 4. The left is a photo randomly shot with a smartphone of a handbag on a chair (left). By finger sketching, the range of the target area is circled, and the system automatically deducts the background and finds the target handbag (middle); finally, by clicking on the search button the user gets the handbag of the same or similar styles on the Internet (right). 


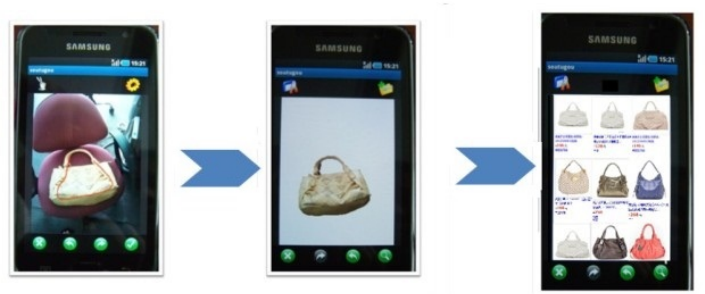

Figure 4. Retrieval of Image with Complex Background

On retrieval efficiency this paper mainly analyzed the cutout time and retrieval time at the mobile client without considering the time consumed by human-computer interaction. Due to difference in phone performance, cutout usually takes 5-10 seconds. For mega-scale data, the retrieval generally takes 0.01 to 0.3 seconds. Classified retrieval by commodity takes shorter time, fully meeting the real-time retrieval demand of general e-commerce platforms.

\section{ACKNOWLEDGMENT}

Mobile platform-based e-commerce is an important trend of the future development and focus of technology research of e-commerce. Image retrieval technology combined with phone camera feature can make up for the lack of intuitiveness in traditional text-based retrieval, which facilitates user selection of products and comparison of prices, significantly enhancing the user shopping experience, thus becomes the core application of the future image retrieval technology.

Based on the image retrieval technology and the characteristics and limitations of mobile client, this paper studied mobile platform-based image retrieval-related technologies, including drag-and-drop input and mobilebased cutout. Through the parallel image retrieval engine, the system supports mega-scale real-time commodity detection and price comparison, thus has a very good practical value.

The follow-up work will continue in-depth study of image feature operators, especially for commodity images with single pattern in order to further improve the validity and accuracy of image retrieval. On feature algorithm, composite analysis of a variety of features will be considered, such as secondary classification-based descriptors of global features and local features. For the clothing commodity images difficult to make linear classification, the possibility of the SVM clustering algorithm-based visual attribute extraction method will be studied; at the same time, the scale of the backstage data will be expanded, more commodity information data will be collected, and the classification will be refined based on commodity type so as to improve retrieval accuracy.

On similarity measurement and high-dimensional search, the more efficient image similarity measure algorithm will be studied, a feedback mechanism will be introduced, and people's subjective judgment and weight analysis will be considered to improve the effectiveness of retrieval. At the same time, the focus will be placed on the local dimensional coding compression algorithm and hardware-based acceleration algorithm in order to optimize the storage needs of the high-dimensional data and improve the retrieval efficiency.

\section{REFERENCE}

[1] Datta R., Joshi D., Li J.,et al., Image Retrieval: Ideas, Influences, and Trends of the New Age[J], ACM Computing Surveys, 40(2), 2008:160.

[2] Riad A.M., Ahmed Atwan A., Hazem M. El-Bakry H.M., et al., An Intelligent Distributed Algorithm for Efficient Web Image Retrieval[J\}, Ineternal Journal of Communications,2009,3(3):63-76.

[3] Zhang J., Liu X.L., Luo J.W. et al., DIRS: Distributed Image Retrieval System Based on MapReduce[C], 2011:93-98.

[4] Zhou D.B., Hu B., Zhang L., High Dimensional Retrieval Base on CUDA[J], Hangzhou Normal University, 2011, 10(5):459-465.

[5] Kruliš M., Skopal T., Lokoč J., Beecks C., Combining CPU and GPU Architectures for Fast Similarity Search[J], Distributed and Parallel Databases, 2012,30(3-4):179-207.

[6] Deselaers T., Keysers D., Ney H., Features for Image Retrieval: An Experimental Comparison[J], Journal of Information Retrieval archive 11(2), 2008:77-107.

[7] Lai C.C., Chen Y.C., A User-Oriented Image Retrieval System Based on Interactive Genetic Algorithm [J], IEEE Transactions on Instrumentation and Measurement, 60(10), 2011:3318-3325.

[8] Deselaers T., Paredes R., Vidal E., et al., Learning Weighted Distances for Relevance Feedback in Image Retrieval[C], The 19th International Conference on Pattern Recognition,2008,1-4.

[9] Liu Y., Zhang D., Lu G., Ma W.Y., A Survey of Content-based Image Retrieval with High-level Semantics[J], Pattern Recognition, 2007, 40(1): 262-282.

[10] GazoPa, http://www.gazopa.com/

[11] Nodari A., Ghiringhelli M., Zamberletti A., A Mobile Visual Search Application for Content Based Image Retrieval in the Fashion Domain[C], 10th International Workshop on Content-Based Multimedia Indexing (CBMI), $2012: 1-6$.

[12] Chinese Patent No. ZL201010155156.9, 2010. 